\title{
A SURVEY ON IOT TECHNOLOGY
}

\author{
Syeda Gauhar Fatima \\ Professor ECE dept, Deccan College of Engineering and Technology, \\ Darussalam, Hyderabad, India \\ Syeda Kausar Fatima \\ Associate Professor ECE dept., \\ Shadan College of Engineering and Technology, Hyderabad, India \\ Dr.K.Anitha Sheela \\ Professor ECE dept, JNTUH, Kukatpally, Hyderabad, India
}

\begin{abstract}
ABTRACT
Internet of Things (IOT) has provided an opportunity to construct powerful industrial system and applications by leveraging the increasing ubiquity of RFID, wireless, mobile and sensor devices. Various industrial IOT applications have been progressively developed and deployed in recent years. Now-a-days, modifying and monitoring plays a main role in our day to day life. Everything we can monitor and control using innovative technologies. Remote access is a ideal feature that came because of high speed internet. The main objective of suggested system is to deliver a technology oriented and low-cost system to make an advanced industry for those who away from their industry and want to control devices.
\end{abstract}

Keywords: Internet of Things (IOT), Server, Raspberry Pi, Webpage, Ethernet, Smart phone etc.

Cite this Article: Syeda Gauhar Fatima, Syeda Kausar Fatima and Dr.K.Anitha Sheela, A Survey on Iot Technology, International Journal of Advanced Research in Engineering and Technology, 10(2), 2019, pp.73-77.

http://iaeme.com/Home/issue/IJARET?Volume=10\&Issue $=2$

\section{INTRODUCTION}

Previously there was a modest manual way of handling machines. However, with the development of technology, new ways are presented for controlling the machines like automation. At the touch of a button, we can contact large amount of information due to ability of computers and the Internet. Everybody wants an affordable and protected way to control their machines from any clever mobile device or Internet connection.

The Internet of Things (IoT) is the network of physical objects or "things" enclosed with electronics, software, sensors and connectivity to enable it to achieve greater value and service by swapping data with the manufacturer, operator or other linked devices. Each thing is 
uniquely identifiable through its embedded computing system but is able to interoperate within the prevailing Internet infrastructure.

Internet of Things is the subsequent big revolution of the world on digitalization of commercializing various modules/products. Everything is connected with the internet, some involves controlling and some involves monitoring the factors from anywhere. The Internet of Things is today's most trending technology that stands together with wearables and robotics.

A printed circuit board (PCB) is the simple part in industry for manufacturing of any electronic product. Etching is main process for developing a PCB. In etching machine, the etchant solution is disseminated over the boards by nozzles and recirculated by pumps. Regulation of the nozzle, temperature and etchant composition gives expected control of etching rates and high manufacturing rate. Etching at ambient temperature might take over an hour, so it is better to heat up the etching solvent to about 35-45 degree celcius. At higher temperatures the etching performance declines, so it is necessary to control the temperature of solvent. So, the proposed system constantly monitors the machine and at a specific situation it will take necessary action.

\section{LITERATURE SURVEY}

$\mathrm{Li} \mathrm{Da} \mathrm{Xu,} \mathrm{Wu} \mathrm{He,} \mathrm{and} \mathrm{Shancang} \mathrm{Li} \mathrm{[1]:} \mathrm{This} \mathrm{paper} \mathrm{reviews} \mathrm{the} \mathrm{existing} \mathrm{research} \mathrm{of} \mathrm{IoT,} \mathrm{key}$ enabling technologies, major IoT applications in industries, and recognizes research trends and challenges. A main contribution of this review paper is that it summarizes the current state-ofthe-art IoT in industries systematically.

AlaaAlhamoud, Felix Ruettiger, Andreas Reinhardt, Frank Englert, Daniel Burgstahler, Doreen Bohnstedt, Christian Gottron and Ralf Steinmetz [2]: This paper presents framework for recognizing energy efficient smart homes based on wireless sensor networks and human movement detection. Their work is based on the idea that most of the user activities at home are related to a set of electrical appliances which are necessary to achieve these activities. Therefore, they show how it is likely to detect the user's present activity by monitoring his finegrained appliance level energy consumption. This relation among activities and electrical appliances makes it possible to identify appliances which could be deteriorating energy at home. Our framework is organized in two components. On one hand, the activity detection framework which is responsible for detecting the user's present activity based on his energy consumption.

Kemal Akkaya, Ismail Guvenc, Ramazan Aygun, Nezih Pala and Abdullah Kadri [3]: This paper surveys the existing works on occupancy monitoring and multi-modal data fusion techniques for smart commercial buildings. The goal is to lay down a framework for future research to exploit the spatio-temporal data achieved from one or more of several IoT devices such as temperature sensors, surveillance cameras, and RFID tags that may be earlier in use in the buildings.

Andrea Zanella, Nicola Bui, Angelo Castellani, LorenzoVangelista, and Michele Zorzi [4]: This paper emphases specifically to an urban IoT system that, while still being quite a broad classification, are described by their specific application domain. Urban IoTs, in fact, are intended to support the Smart City vision. This aims at misusing the most advanced communication technologies to support added-value facilities for the management of the city and for the citizens. This paper hence offers a complete survey of the enabling technologies, protocols, and architecture for an urban IoT.

Ala Al-Fuqaha, Mohsen Guizani, Mehdi Mohammadi, Mohammed Aledhari, and Moussa Ayyash [5]: This paper provides an overview of the Internet of Things (IoT) with emphasis on enabling technologies, protocols, and application issues. The IoT is enabled by the latest developments in RFID, smart sensors, communication technologies, and Internet protocols. The basic premise is to have smart sensors collaborate directly without human involvement to 
deliver a new class of applications. The current revolution in Internet, mobile, and machinetomachine (M2M) technologies can be seen as the first phase of the IoT. In the coming years, the IoT is expected to bridge diverse technologies to enable new applications by connecting physical objects together in support of intelligent decision making. This paper provides a horizontal overview of the IoT. Then give an overview of some technical details that pertain to the IoT enabling technologies, protocols, and applications. Compared to other survey papers in the field, our objective is to provide a more thorough summary of the most relevant protocols and application issues.

\section{SUGGESTED WORK}

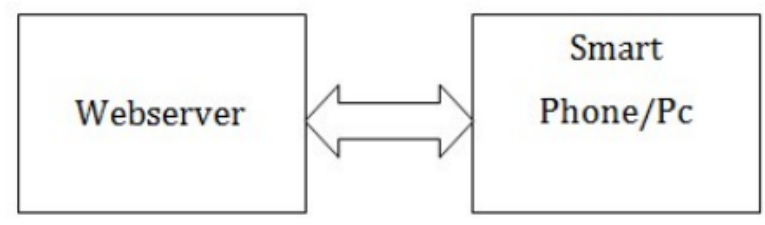

Figure 1 Server block diagram

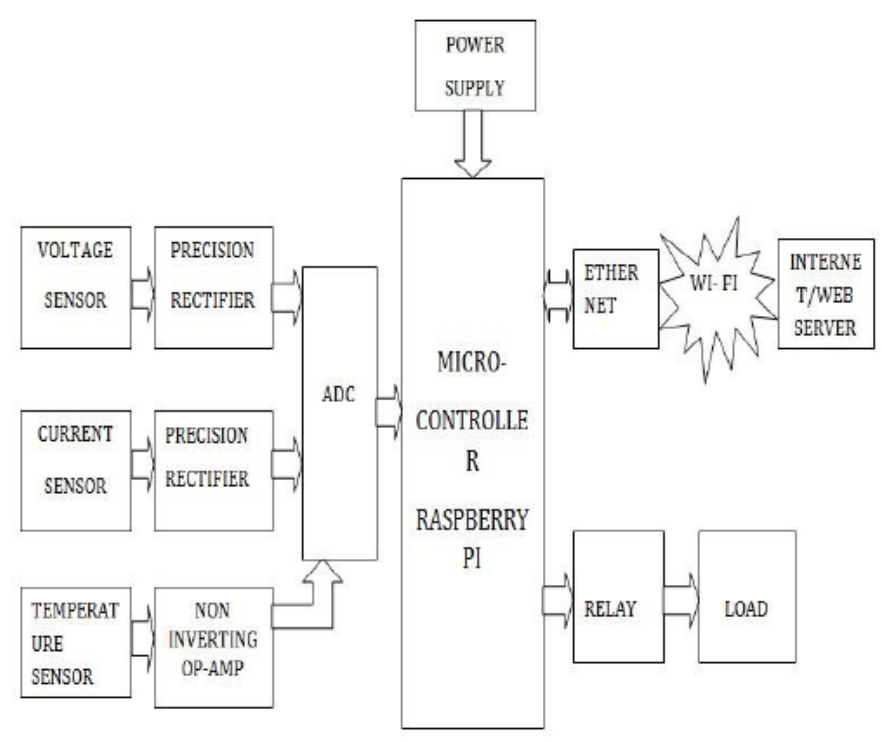

Figure 2 Client block diagram

The complete system is composed of two parts: Server and Client. When power supply is given, sensors starts sensing the consistent parameters. The data collected by sensors is trained and amplified to interface it with RaspberryPi system. At the same time the sensed values are uploaded onto webpage. The authorized person can access the data from any place at any time, monitor the factors and control the load through IOT successfully.

An accredited person can login by using username and password. If username and password is correct then and then only user can monitor and control the machine. Maintenance is one of the major concerns in industry. The voltage and current of induction motor can be designed by using voltage sensor and current sensor. By using this factors power consumption can be calculated and according to that indication is given. Green signal for no maintenance, orange signal for maintenance is required within 10-15 days, red signal for maintenance require. When power exceeds particular level then the motor gets automatically turn off. Temperature sensor is used to monitor the temperature of solvent and control the heater in the machine. When 
temperature goes away from particular temperature the heater gets spontaneously turned off. The Webpage is used to monitor and control of machine using the data collected by Raspberry Pi platform.

Raspberry- Pi: These boards contain the ARM11 IC. ARM11 processor (Raspberry Pi) plays a key role in monitoring the system. Low-power consumption ARM11 processor (Raspberry Pi) operating at $3.3-5 \mathrm{~V}, 50 \mathrm{uA}-1 \mathrm{~A}$ is designed and mounted on a PCB along with reset circuit and clock circuit. ARM11 is 32-bit processor with RISC architecture and having 40 GPIO with 8GB SD Card and 512 Bytes of RAM associated with this raspberry pi.

Temperature Sensor: Temperature sensor is used to monitor the temperature of chemical, which is used for etching purpose. When temperature exceeds particular temperature then the heater gets automatically off.

Current sensor: Current transformer is sensor used to linearly step down the sensor to a lower level compatible with measurement instrumentation. The core of a current transformer is toroidal, or ringed, in shape with opening in the center. The number of wire winding around the core dictates the step-down ratio, between the current in measured line, and the current output linked to the instrumentation.

Voltage sensor: Voltage transformer is a sensor used to linearly step down the sensor to a lower level compatible with measurement instrumentation. The number of wire winding around the core dictates the step down ratio, between the voltage in measured line, and the voltage output linked to the instrumentation.

Webpage: HTML is a specific type of universal language used for decorating a web page.HTML stands for Hypertext Markup Language. Hypertext is the text that has been used up with extra specifications such as formatting, Image multimedia etc.

Relay: Relay is a device which allows low power circuit to switch a relatively high current/voltage and controlling the actions achieved. Designing this on PCB we are connecting the appliances like bulb, DC motor etc.

\section{CONCLUSION}

The monitoring and controlling of machines in industry has become a main problem. By studying all the above reference papers most of the work done related to home automation. The proposed system take few parameters related to industry and deliver facility related to that The recommended system enables us to monitor and control machines from remote places as well as gives facts regarding the maintenance. This kind of system useful because Human can make mistakes and forgot to switch off the machine at specific situation.

\section{REFERENCES}

[1] Li Da Xu, Wu He, and Shancang Li,"Internet of Things in Industries: A Survey" IEEE transactions on industrial informatics, vol. 10, no. 4, November 2014.

[2] Alaa Alhamoud, Felix Ruettiger, Andreas Reinhardt, Frank Englert, Daniel Burgstahler, Doreen Bohnstedt, Christian Gottron and Ralf Steinmetz," SMARTENERGY.KOM: An Intelligent System for Energy Saving in Smart Home", 3rd IEEE international workshop on global trends 2014.

[3] Kemal Akkaya, Ismail Guvenc, Ramazan Aygun, Nezih Pala, Abdullah Kadri,” IOT-based Occupancy Monitoring Techniques for Energy-Efficient Smart Buildings”,2015 IEEE wireless communication and networking conference.

[4] Andrea Zanella, Nicola Bui, Angelo Castellani,Lorenzo Vangelista, and Michele Zorzi, "Internet of Things for Smart Cities" IEEE Internet of things journal, Vol. 1, No. 1 , FEBRUARY2014. 
[5] Ala Al-Fuqaha, Mohsen Guizani, Mehdi Mohammadi,Mohammed Aledhari, and Moussa Ayyash,"Internet of Things: A Survey on EnablingTechnologies, Protocols, and Applications",IEEE communication surveys \& tutorials, vol. 17, no. 4, fourth quarter 2015.

[6] Nagender Kumar Suryadevara, Subhas Chandra Mukhopadhyay, Sean Dieter Tebje Kelly, and Satinder Pal Singh Gill," WSN-Based Smart Sensors and Actuator for Power Management in Intelligent Buildings" IEEE/ASME transactions on mechatronics, vol. 20, no. 2, april 2015.

[7] Dan D. Koo, John J. Lee, Aleksei Sebastiani, and Jonghoon Kim,” An Internet-of-Things (IoT) system development and implementation for bathroom safety enhancement"International Conference on Sustainable Design, Engineering and Construction, Sciencedirect Procedia Engineering 145 ( 2016 ) 396 - 403.

[8] Vinay M, Shivashankar s k,"Monitoring And Controlling Of Smart Equipments In Manufacturing Industry Using Iot Applications",International Journal of Technical Research and Applications e-ISSN: 2320-8163, www.ijtra.com Volume 4, Issue 3 (MayJune, 2016), PP. 96-100.

[9] Dr. Chalasani Srinivas and Mohan Kumar.Ch. Toxic GAS Detection and Monitoring utilizing Internet of Things. International Journal of Civil Engineering and Technology, 8(12), 2017, pp. 614-622

[10] Bhavana Godavarthi, G Manu, A Sudhakar and Paparao Nalajala, Underground Cable Acoustic Fault Route Tracking and Distance Identifying In Coal Mine Using Internet of Things, International Journal of Civil Engineering and Technology, 8(8), 2017, pp. 762 771.

[11] Dr.Chitra, Dr.K.Uma and M.Basha Khaja, Wireless Health Acquisition System Using Internet of Things, International Journal of Mechanical Engineering and Technology, 9(11), 2018, pp. 902-907.

[12] Carlos M. S. Rodrigues, Bruno S. L. Castro, A Vision of Internet of Things in Industry 4.0 with ESP8266. International Journal of Electronics and Communication Engineering and Technology, 9(1), 2018, pp. 1-12

[13] Kaleel Ahmed A and Prof. Dr. C.B. Senthil Kumar, Correlating Internet of Things. International Journal of Management, 8 (2), 2017, pp. 68-76.

[14] Chitra Kiran N and Dr. G. Narendra Kumar, Efficient M-Commerce Marketing Application Based Internet of Things Using Near Field Communication, International Journal of Computer Engineering and Technology (IJCET), Volume 3, Issue 3, October-December (2012), pp. 15-22

[15] Hina ruqsar, Chandana. R, Nandini. R and Dr. T P Surekha, Internet of Things (Iot) Based Real Time Gas Leakage Monitoring and Controlling, International Journal of Electronics and Communication Engineering and Technology (IJECET), Volume 5, Issue 8, August (2014), pp. 208-214 